\title{
Effect of 3-Year Weight History on Blood Pressure: The Atherosclerosis Risk in Communities Study
}

\author{
Kimberly P. Truesdale ${ }^{1}$, June Stevens ${ }^{1,2}$, and Jianwen Cai ${ }^{3}$ \\ ${ }^{1}$ Department of Nutrition, School of Public Health, University of North Carolina at Chapel Hill, \\ Chapel Hill, North Carolina, USA \\ ${ }^{2}$ Department of Epidemiology, School of Public Health, University of North Carolina at Chapel Hill, \\ Chapel Hill, North Carolina, USA \\ ${ }^{3}$ Department of Biostatistics, School of Public Health, University of North Carolina at Chapel Hill, \\ Chapel Hill, North Carolina, USA
}

\begin{abstract}
The objective of this study was to examine the effect of weight history on blood pressure. Extant data from the Atherosclerosis Risk in Communities (ARIC) study were used to compare blood pressure in women $(n=5,675)$ and men $(n=4,893)$ with different 3-year weight histories, but similar current BMI. We used mixed models regression adjusted for ethnicity, age, education, field center, smoking, alcohol consumption, antihypertensive medications, interval length, and BMI at follow-up. We also examined associations between 3-year weight history and blood pressure within weight status categories (normal weight $\left(\geq 18.5\right.$ to $<25.0 \mathrm{~kg} / \mathrm{m}^{2}$ ), overweight $(\geq 25.0$ to $\left.<30.0 \mathrm{~kg} / \mathrm{m}^{2}\right)$, and obese $\left.\left(\geq 30.0 \mathrm{~kg} / \mathrm{m}^{2}\right)\right)$. We found weight history affected both systolic and diastolic blood pressures. Compared to men at the same BMI who had maintained their weight, men who had experienced a $10 \%$ weight gain over the previous 3 years had systolic and diastolic blood pressures that were 2.6 and $1.9 \mathrm{~mm} \mathrm{Hg}$ higher, respectively $(P<0.001$ for both).

Associations in women were in the same direction, but smaller at 0.9 and $0.6 \mathrm{~mm} \mathrm{Hg}(P<0.001)$. With the exception of diastolic blood pressure in normal weight women, we found no significant interactions between weight change and current weight status. In conclusion, some of the variation in blood pressure among individuals at the same BMI may be due to weight change history.

Effects of 3-year weight change history appear to be stronger and more consistent in men than in women, and generally similar regardless of current weight status.
\end{abstract}

\section{INTRODUCTION}

There is a positive association between body weight and blood pressure, with hypertension being more prevalent among obese (BMI $\geq 30.0 \mathrm{~kg} / \mathrm{m}^{2}$ ) and overweight ( $\geq 25.0$ to $<30.0 \mathrm{~kg}$ / $\mathrm{m}^{2}$ ) than normal weight $\left(\geq 18.5\right.$ to $\left.<25.0 \mathrm{~kg} / \mathrm{m}^{2}\right)$ adults (1). A positive association between weight gain and blood pressure has also been well documented (2-8). However, research on weight cycling (repeatedly losing and gaining weight) has yielded inconsistent findings (9), and little is known about the impact of weight history on blood pressure. It seems plausible that adults with a history of higher weight might maintain higher blood pressure values when compared to adults with the same current BMI.

\footnotetext{
(C) 2008 The Obesity Society

Correspondence: Kimberly P. Truesdale (Kim_Truesdale@unc.edu).

Disclosure

The authors declared no conflict of interest.
} 
Our group has recently shown that, compared to normal weight adults who had maintained their weight for 3 years, normal weight adults who were heavier 3 years earlier (i.e., had lost weight) had similar glucose, high-density lipoprotein-cholesterol, and triglycerides levels, and more favorable total and low-density lipoprotein-cholesterol levels (10). Thus, it appeared that heavier persons who became normal weight acquired levels of glucose and lipids that were not less favorable than those of weight maintainers with similar current BMI. Therefore, they were not penalized for being heavier earlier in their lives concerning their glucose and lipid levels.

The objective of this study was to examine the effects of weight history on blood pressure in adults with similar current BMI. Specifically, we will determine whether (i) systolic and diastolic blood pressures differed in women and men with different 3-year weight histories but similar current BMI; and (ii) the relationships of blood pressure with weight history differed by current weight status categories (normal weight, overweight, or obese). To our knowledge, this is the first study to examine these issues systematically.

\section{MATERIALS AND METHODS}

\section{Study participants}

The Atherosclerosis Risk in Communities (ARIC) study is a prospective investigation of the natural history and etiology of atherosclerosis and cardiovascular disease in four US communities: Forsyth County, North Carolina; Jackson, Mississippi; the northwestern suburbs of Minneapolis, Minnesota; and Washington County, Maryland. Baseline data were collected between 1987 and 1989 on 15,792 adults 45-64 years of age. Three follow-up visits took place at $\sim 3$-year intervals. The follow-up rates among participants who were alive at each visit were $92.7,85.6$, and $80.8 \%$ at visits 2,3 , and 4 , respectively. The details of the study design have been described previously (11). This study was approved by the Institutional Review Boards at each field center and this analysis was approved by the University of North Carolina at Chapel Hill Public Health Institutional Review Board on research involving human subjects.

\section{Measures, covariates, and exclusions}

The participants were weighed in a scrub suit without shoes to the nearest pound using a beam balance scale at all visits. The participant's height (without shoes) was measured to the nearest centimeter using a metal ruler attached to a wall and a standard triangular headboard. Participants were classified as normal weight, overweight, or obese based on their calculated BMI (weight $(\mathrm{kg}) /$ height $\left(\mathrm{m}^{2}\right)$ ) at the end of the 3-year interval. Below, the beginning of each interval is referred to as previous or time 1 and the end of the interval as current or time 2. For each 3-year interval, the percent weight change was calculated using the weight at time 1 as the denominator. We created seven weight change categories. Weight maintenance (referent) was defined using a recently proposed definition of $\pm 3.0 \%$ (12). Three categories for weight gain and weight loss were defined as follows: $\geq 10.0 \%, \geq 5.0$ to $<10.0 \%$, and $>3.0$ to $<5.0 \%$.

Blood pressure was measured three times after a 5-min rest using a random zero sphygmomanometer on the right arm of the seated participant. The average of the last two measures was used for the analysis. Participants' education level was categorized as having less than a high school education, high school graduate, or at least some college. At each visit, participants self-reported their cigarette smoking and alcohol beverage consumption status (current, former, or never) and whether they were taking antihypertensive medication.

Following standard ARIC protocol, we excluded the 55 African Americans from Washington County, Maryland or Minneapolis, Minnesota and the 48 participants who 
classified their ethnicity as other than White or African American because they were too small in number to allow ethnic and field center-specific analyses. A race-center variable was created because all field centers did not have both African Americans and whites in the analysis data set.

For each 3-year interval (visits 1-2, 2-3, and 3-4), we excluded participants whether they were missing either visit $(n=1,432,3,093$, and 4,516); missing BMI at either visit ( $n=50$, 43 , and 44$)$; underweight $\left(<18.5 \mathrm{~kg} / \mathrm{m}^{2}\right)$ at either visit $(n=162,150$, and 127$)$; missing blood pressure at time $2(n=5,5$, and 6); taking antihypertensive medications at time 1 ( $n=$ $4,188,3,971$, and 3,873); or missing pertinent variables ( $n=68,34$, and 62), respectively. After exclusions, we created a "stacked" data set such that, each participant $(5,675$ women and 4,893 men) had between 1 and 3 repeated observations $(N=13,550$ women and 11,688 men).

\section{Statistical analysis}

Repeated measures regression (PROC MIXED procedure with compound symmetry in SAS software version 9.1; SAS Institute, Cary, NC) was used to construct longitudinal models of 3 -year weight history. All models were stratified by gender. The full model adjusted for race-center and education; age, BMI, cigarette smoking status, alcohol beverage consumption status, and antihypertensive medication usage at time 2 ; and elapsed time between visits. Adjusted mean systolic and diastolic blood pressures were calculated for each weight history category using the LSMEANS option and the gender-specific population prevalence or mean of each covariate except current BMI. BMI was set approximately at the mean $\left(27.5 \mathrm{~kg} / \mathrm{m}^{2}\right)$ to focus our findings on the effects of weight history, rather than current weight. In order to further investigate these patterns, we stratified by current weight status category. Current BMI was set to $23.0,27.0$ or $34.0 \mathrm{~kg} / \mathrm{m}^{2}$ for the normal weight, overweight, and obese strata, respectively.

We used continuous percent weight change to predict the adjusted increase in blood pressure given a $10 \%$ of weight gain. In order to determine whether the relationship between current blood pressure and 3-year weight change history differed by current weight status, we examined the interaction between continuous weight change history and current weight status category.

\section{RESULTS}

We found significant $(P<0.05)$ weight history category by gender interactions; however, within gender, there were no weight history by race interactions. Results were similar using continuous weight change history; therefore, all results are presented stratified by gender.

The unadjusted frequency distributions and mean values of selected demographic characteristics are shown by weight history categories for women and men (Table 1 ). The weight maintenance group had the largest number of observations among women $(n=$ $5,909)$ and men $(n=6,241)$. Compared to weight maintenance, a history of weight loss tended to have lower current mean BMI and history of weight gain had higher current mean BMI. Antihypertensive medication usage was less prevalent among weight maintainers than either weight losers or weight gainers (except women who gained $>3.0$ to $<5.0 \%$ ).

Table 2 shows the age and race-center adjusted blood pressure levels at time 1 (before weight change history). In general, compared to weight maintainers, women and men with a previous higher weight had higher systolic and diastolic blood pressure levels at time 1 and participants with a previous lower weight had lower blood pressure levels. Patterns tended to be similar after stratifying by current weight status. 


\section{Analysis of weight history categories}

Figure 1 shows the mean current systolic blood pressure levels in women with seven different 3-year weight histories. We set the $\mathrm{x}$ axis to cross the $\mathrm{y}$ axis at the mean blood pressure level for weight maintainers (referent group). Overall, in women (Figure 1a), weight history appeared to have little impact on systolic blood pressure. After stratifying by current weight status (Figures 1b-d), systolic blood pressure tended to be similar across the seven 3-year weight history categories.

In men (Figure 2), difference across the seven 3-year weight history categories was more prominent. Overall, compared to men with a history of weight maintenance, men with a history of weight loss had significantly lower systolic blood pressure levels and men with a history of weight gain had higher levels. Patterns similar to those seen in the overall analysis were evident in normal weight (Figure 2b) and overweight (Figure 2c) men, but not apparent in obese men (Figure 2d).

Figures 3 (women) and 4 (men) shows similar plots with diastolic rather than systolic blood pressure as the outcome. In general, participants who had lost weight had lower diastolic blood pressure and participants who had gained weight had higher blood pressure than participants who maintained their weight, although differences were not always statistically significant. The stratified results in women (Figures 3b-d) and men (Figures $4 b-d$ ) showed the similar patterns, but differences were more often apparent and statistically significant in men.

\section{Analyses of continuous weight change}

Table 3 shows the associations between continuous percent weight change and blood pressure. Compared to men in the same weight status category who had maintained their weight, men who had experienced a $10 \%$ weight gain over the previous 3 years had systolic and diastolic blood pressures that were 2.6 and $1.9 \mathrm{~mm} \mathrm{Hg}$ higher, respectively $(P<0.001$ for both). Associations in women were in the same direction, but substantially smaller at 0.9 and $0.6 \mathrm{~mm} \mathrm{Hg}(P<0.001)$. The magnitude of the changes in systolic and diastolic blood pressures was similar in men regardless of their current weight status. In contrast, we found a significant interaction between continuous percent weight change and current weight status among women for diastolic blood pressure $(P=0.028)$. The association between weight history and diastolic blood pressure was smaller in normal weight women than in overweight and obese women. Similar effect modification was found when weight history was examined in categories.

\section{DISCUSSION}

The purpose of this work was to examine the effects of weight history on blood pressure in adults with similar current BMIs. The two concepts of previous BMI and previous weight change cannot be entirely separated in an analysis such as the one presented here. When comparing individuals who currently have a similar BMI, those who were previously of a higher weight, had to have had weight loss; and similarly individuals who were previously of a lower weight had to have had weight gain. The literature indicates that having a high $\mathrm{BMI}$ is associated with having a higher blood pressure, and that weight loss is associated with a decline in blood pressure. We found that blood pressure levels adjusted for current BMI were lower if the participant had a history of weight loss (previously higher BMI), and blood pressure was higher if the participant had a history of weight gain (previously lower $\mathrm{BMI})$. The direction of our findings, given similar current BMI, lead us to conclude that BMI 3 years prior had little impact on blood pressure, whereas the experience of either weight gain or weight loss over a 3 -year period did impact blood pressure. The estimates 
varied slightly and similar patterns were observed when we excluded adults currently on antihypertensive medication.

There is strong evidence that blood pressure improves with weight loss over a range of levels in BMI $(2-7,13)$. In the Trials of Hypertension Prevention, researchers found a direct association between the amount of weight loss and blood pressure reduction at 36 months (8). Prospective cohort studies have also found that blood pressure and hypertension prevalence decreases with weight loss (14-16). In addition, several researchers have found a positive associations between weight gain and obesity with blood pressure and hypertension (2,14,17-19). In the Iowa Women's Health Study, French et al. (1996) found that women with weight status patterns described as weight loss regain; weight gain maintenance; or continuous weight gain were at increased odds of developing hypertension regardless of their weight status at the age of 18 when compared to weight stable women who were normal weight at age 18 (ref. 19). Weight stable women who were overweight at age 18 were also at increased risk.

Different from the work presented here, French et al. did not standardize results from participants with different weight histories to a common weight at follow-up. In this study, when comparing adults with different weight histories we found the results varied by gender. Both current systolic and diastolic blood pressures were lower when weight 3 years earlier had been heavier. The findings were in a consistent direction regardless of current weight status category, although the size of the effect on diastolic blood pressure was smaller in normal weight women.

We found that systolic and diastolic blood pressures increased in men and women with weight gain; however, the magnitude of the change was substantially larger in men compared to women given the same percentage of weight change. Similar trends were also observed among men for systolic and diastolic blood pressures regardless of their current weight status category. In contrast, the magnitude of the changes in diastolic blood pressure associated with weight gain was smaller in normal weight women than overweight and obese women.

Cross-sectional analysis of the National Health and Nutrition Examination Survey III data has shown that systolic and diastolic blood pressures were higher among young men than women. In addition, hypertension is more prevalent among men than women until age 59, then it is more prevalent among women (20). Wing and Jeffery (1995) examined whether the effect of weight loss on blood pressure and other cardiovascular disease risk factors were comparable in men and women who participated in an 18-month behavioral weight loss program (21). They found men had a significantly larger decreases in body weight, waist circumference, waist-to-hip ratio, and percent body fat compared to women. Wing and Jeffrey hypothesized that this was because men and women were often given the same calorie allowances when on a weight loss diet despite different calorie requirements, thus creating a larger deficit among men. In this study, the mean weight change among $\geq 5.0$ to $<10.0 \%$ weight losers were similar between women $(-7.0 \%)$ and men $(-6.9 \%)$. In contrast, the change in systolic $(0.1 \mathrm{~mm} \mathrm{Hg}$ vs. $-2.7 \mathrm{~mm} \mathrm{Hg})$ and diastolic $(-3.1 \mathrm{~mm} \mathrm{Hg}$ vs. -4.1 $\mathrm{mm} \mathrm{Hg}$ ) blood pressure was smaller in women than men. The gender differences observed in the current study could be due to differences in weight loss methods (dieting or exercising).

In a review by LaRosa, the author concluded that lifestyle interventions appear to be more effective at reducing risk in men than in women (22); however, that review focused on lipids not blood pressure. The opposite findings were found in a 2000 review of exercise training among hypertensive adults (23). The authors found although exercise training decreased 
systolic and diastolic blood pressures, the reduction was larger among women compared to men for systolic (14.7 $\mathrm{mm} \mathrm{Hg}$ vs. $8.7 \mathrm{~mm} \mathrm{Hg}$ ) and diastolic (10.5 mm Hg vs. $7.8 \mathrm{~mm} \mathrm{Hg}$ ) blood pressures, respectively. The conflicting results may be explained by the different population samples. Hagberg et al. (23) reviewed only studies among hypertensive adults whereas this study included both normotensive and hypertensive adults.

Potential limitations of this study are the lack of information on the intentionality of weight changes and weight cycling during the 3-year interval. Data are not available on weight changes between clinic visits. It is possible that the magnitude and direction of these changes may differ by gender. Analysis of the 1989 National Health Information Survey determined that among adults who lost weight, $60 \%$ reported that the weight loss was intentional (24). Unintentional weight loss has been associated with poorer health status and smoking $(24,25)$. In an effort to eliminate the impact of large weight changes that could be the result of illness, we repeated the analysis (data not shown) after excluding all weight change $>20 \%$. We found similar results; however, it is still possible that illness-related weight changes affected the study findings. Changes in diet and physical activity could also alter or explain the study findings. We were unable to examine these changes over all 3-year intervals because diet and physical activity were not assessed at every examination in ARIC. Using a subset of the data, we re-analyzed the data controlling for physical activity, type of diet, and other dietary intake variables and found similar results (data not shown). In addition, the mean current BMI tended to differ significantly across the weight history categories. To ensure that this did not bias our findings, we repeated the analysis after restricting the datasets such that the mean BMI was similar across the seven weight history categories. This maneuver did not substantially change our estimates or conclusions (data not shown).

Although clinical trials can produce strong evidence, it is difficult to conduct clinical trials of long-term weight loss because it is very hard for participants to actually lose weight and maintain weight loss over a period of time as long as 3 years. In addition, there could be ethical issues involved in randomizing participants to gain weight. This study design did provide a feasible mechanism for observing relatively long-term effects of weight history. The 3-year interval studied here was longer than that in many weight loss studies. In addition, this study used a community-based sample, and this increased the generalizability. However, we excluded adults taking antihypertensive medication at time 1, and this limits the generalizability of our results. Another important strength of this study was the availability of measured weights and blood pressure in the same participants over a series of examinations.

In contrast to our hypothesis, this study found that adults who were heavier 3 years prior tended to have similar (women) or more favorable (men) blood pressure levels as adults with the same BMI who maintained their weight. The patterns across the 3-year weight history categories were similar regardless of current weight status, and this has important public health implications given the current overweight and obesity prevalence rates. To our knowledge, this is the first study to compare blood pressure levels among women and men with different weight histories but similar BMI levels. Additional research is needed to determine how long the changes in blood pressure associated with weight change endure and if risk of cardiovascular events follow trends similar to those assessed here for blood pressure.

\section{Acknowledgments}

The Atherosclerosis Risk in Communities (ARIC) study is carried out as a collaborative study supported by National Heart, Lung, and Blood Institute contracts N01-HC-55015, N01-HC-55016, N01-HC-55018, N01- 
HC-55019, N01-HC-55020, N01-HC-55021, and N01-HC-55022. The authors thank the staff and participants of the ARIC study for their important contributions.

\section{References}

1. Gregg EW, Cheng YJ, Cadwell BL, et al. Secular trends in cardiovascular disease risk factors according to body mass index in US adults. JAMA. 2005; 293:1868-1874. [PubMed: 15840861]

2. National Heart Lung and Blood Institute. Clinical guidelines on the identification, evaluation, and treatment of overweight and obesity in adults-the evidence report. Obes Res. 1998; 6(Suppl 2): 51S-209S. [PubMed: 9813653]

3. Blackburn G. Effect of degree of weight loss on health benefits. Obes Res. 1995; 3(Suppl 2):211S216S. [PubMed: 8581779]

4. Klein S. Outcome success in obesity. Obes Res. 2001; 9(Suppl 4):354S-358S. [PubMed: 11707565]

5. Goldstein DJ. Beneficial health effects of modest weight loss. Int J Obes. 1992; 16:397-415.

6. Mertens IL, Van Gaal LF. Overweight, obesity, and blood pressure: the effects of modest weight reduction. Obes Res. 2000; 8:270-278. [PubMed: 10832771]

7. Pi-Sunyer FX. Short-term medical benefits and adverse effects of weight loss. Ann Intern Med. 1993; 119:722-726. [PubMed: 8363205]

8. Stevens VJ, Obarzanek E, Cook NR, et al. Long-term weight loss and changes in blood pressure: results of the Trials of Hypertension Prevention, phase II. Ann Intern Med. 2001; 134:1-11. [PubMed: 11187414]

9. National Task Force on the Prevention and Treatment of Obesity. Weight cycling. JAMA. 1994; 272:1196-1202. [PubMed: 7741844]

10. Truesdale KP, Stevens J, Cai J. The effect of weight history on glucose and lipids: the Atherosclerosis Risk in Communities Study. Am J Epidemiol. 2005; 161:1133-1143. [PubMed: 15937022]

11. ARIC Investigators. The Atherosclerosis Risk in Communities (ARIC) Study: design and objectives. the ARIC investigators. Am J Epidemiol. 1989; 129:687-702. [PubMed: 2646917]

12. Stevens J, Truesdale KP, McClain JE, Cai J. The definition of weight maintenance. Int J Obes. 2006; 30:391-399.

13. de Leiva A. What are the benefits of moderate weight loss? Exp Clin Endocrinol Diabetes. 1998; 106(Suppl 2):10-13. [PubMed: 9792475]

14. Droyvold WB, Midthjell K, Nilsen TI, Holmen J. Change in body mass index and its impact on blood pressure: a prospective population study. Int J Obes. 2005; 29:650-655.

15. Moore LL, Visioni AJ, Qureshi MM, et al. Weight loss in overweight adults and the long-term risk of hypertension: the Framingham study. Arch Intern Med. 2005; 165:1298-1303. [PubMed: 15956011]

16. Huang Z, Willett WC, Manson JE, et al. Body weight, weight change, and risk for hypertension in women. Ann Intern Med. 1998; 128:81-88. [PubMed: 9441586]

17. Juhaeri, Stevens J, Chambless LE, et al. Associations between weight gain and incident hypertension in a bi-ethnic cohort: the Atherosclerosis Risk in Communities Study. Int J Obes. 2002; 26:58-64.

18. Field AE, Byers T, Hunter DJ, et al. Weight cycling, weight gain, and risk of hypertension in women. Am J Epidemiol. 1999; 150:573-579. [PubMed: 10489996]

19. French SA, Jeffery RW, Folsom AR, McGovern P, Williamson DF. Weight loss maintenance in young adulthood: prevalence and correlations with health behavior and disease in a populationbased sample of women aged 55-69 years. Int J Obes. 1996; 20:303-310.

20. Burt VL, Whelton P, Roccella EJ, et al. Prevalence of hypertension in the US adult population. Results from the Third National Health and Nutrition Examination Survey, 1988-1991. Hypertension. 1995; 25:305-313. [PubMed: 7875754]

21. Wing RR, Jeffery RW. Effect of modest weight loss on changes in cardiovascular risk factors: are there differences between men and women or between weight loss and maintenance? Int J Obes. 1995; 19:67-73. 
22. LaRosa JC. Lipids and cardiovascular disease: do the findings and therapy apply equally to men and women? Womens Health Issues. 1992; 2:102-113. [PubMed: 1617306]

23. Hagberg JM, Park JJ, Brown MD. The role of exercise training in the treatment of hypertension: an update. Sports Med. 2000; 30:193-206. [PubMed: 10999423]

24. Meltzer AA, Everhart JE. Unintentional weight loss in the United States. Am J Epidemiol. 1995; 142:1039-1046. [PubMed: 7485049]

25. French SA, Jeffery RW, Folsom AR, et al. Relation of weight variability and intentionality of weight loss to disease history and health-related variables in a population-based sample of women aged 55-69 years. Am J Epidemiol. 1995; 142:1306-1314. [PubMed: 7503051] 

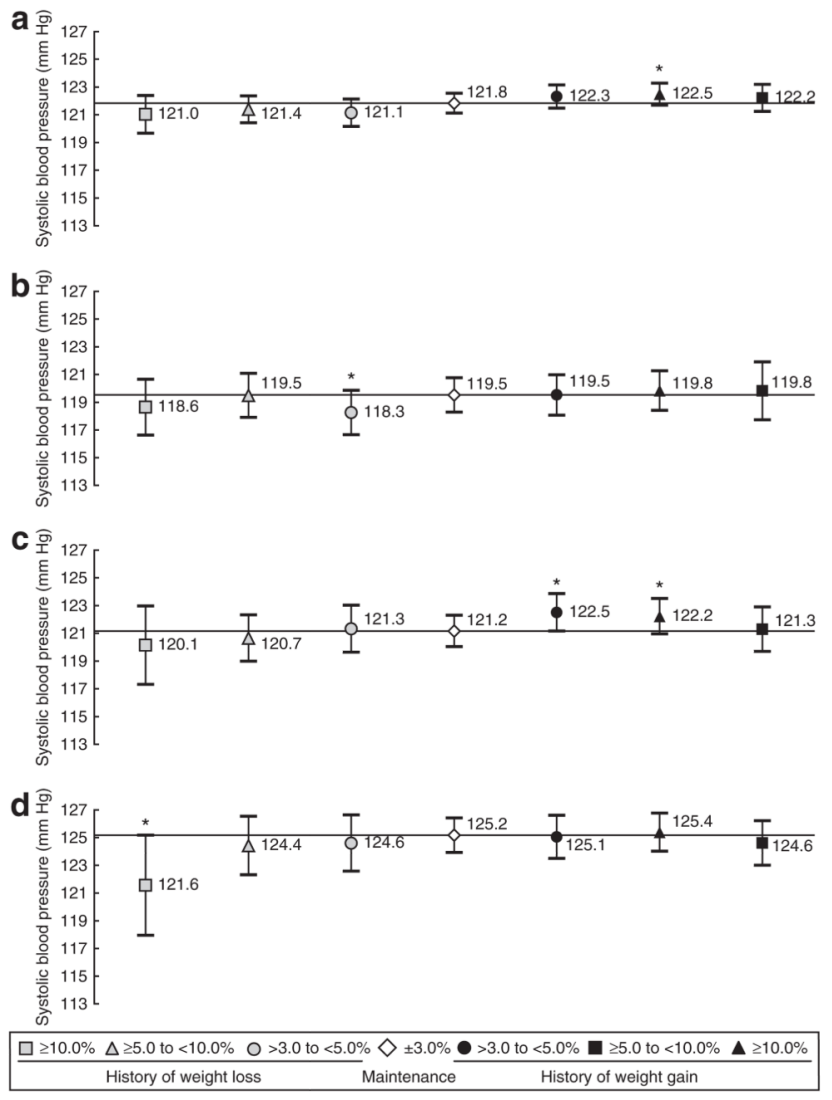

Figure 1.

Current mean systolic blood pressure (and 95\% confidence intervals) among women with different 3-year weight histories, (a) overall and by current weight status ((b) normal weight; (c) overweight; or (d) obese), the Atherosclerosis Risk in Communities Study, 1987-1998. Models were adjusted for race-center and education; age, BMI, cigarette smoking status, alcohol beverage consumption status, and antihypertensives at time 2; and elapsed time between visits. *Significantly different $(P<0.05)$ from weight maintainers. 
a

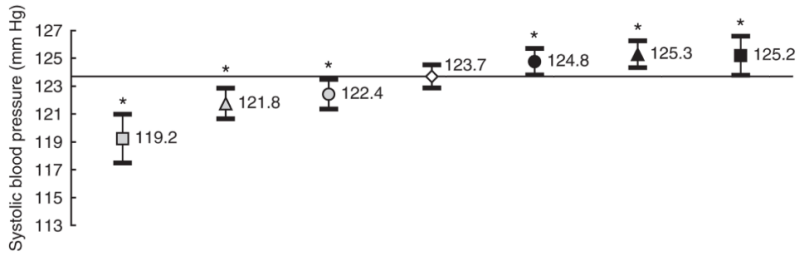

b

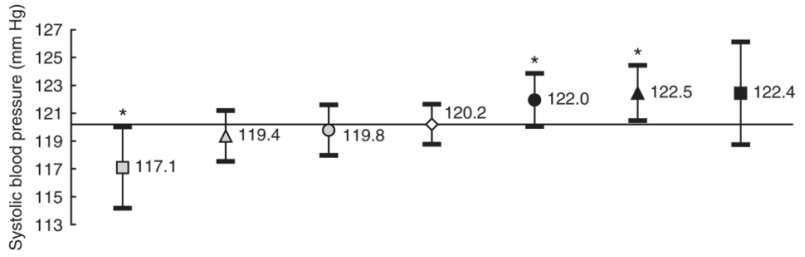

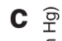
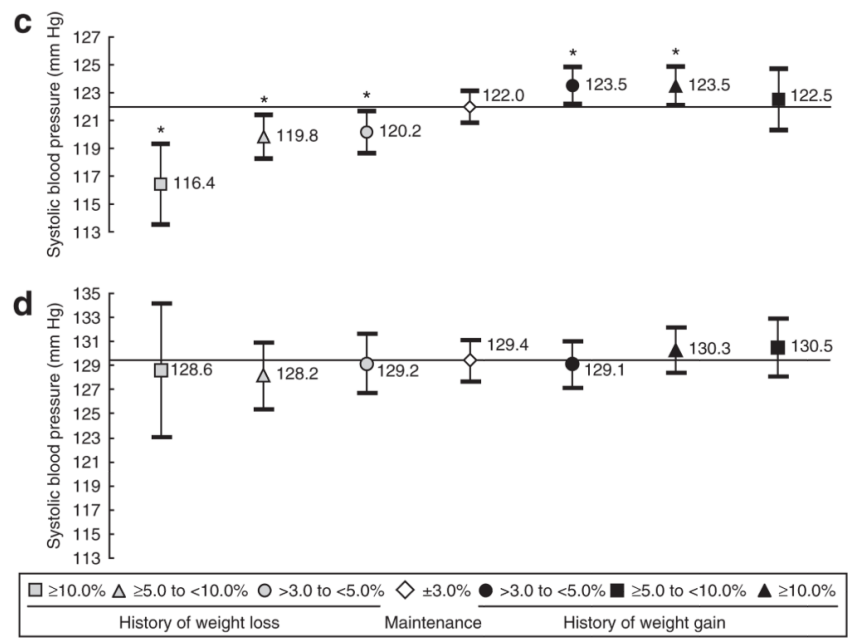

Figure 2.

Current mean systolic blood pressure (and 95\% confidence intervals) among men with different 3-year weight histories, (a) overall and by current weight status ((b) normal weight; (c) overweight; or (d) obese), the Atherosclerosis Risk in Communities Study, 1987-1998. Models were adjusted for race-center and education; age, BMI, cigarette smoking status, alcohol beverage consumption status, and antihypertensives at time 2; and elapsed time between visits. *Significantly different $(P<0.05)$ from weight maintainers. 

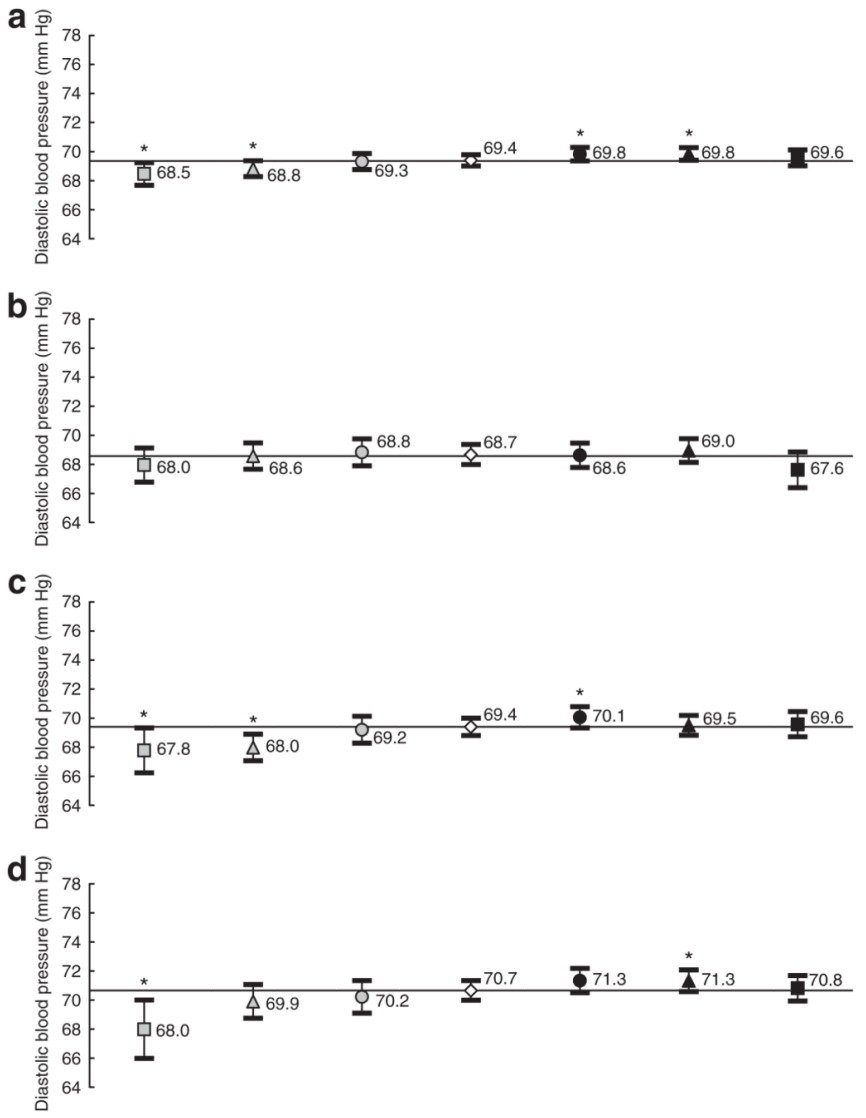

$\square \geq 10.0 \% \Delta \geq 5.0$ to $<10.0 \%$ O $>3.0$ to $<5.0 \% \diamond \pm 3.0 \% \quad>3.0$ to $<5.0 \%$ a $\geq 5.0$ to $<10.0 \% \Delta \geq 10.0 \%$ History of weight loss Maintenance History of weight gain

Figure 3.

Current mean diastolic blood pressure (and 95\% confidence intervals) among women with different 3-year weight histories, (a) overall and by current weight status ((b) normal weight; (c) overweight; or (d) obese), the Atherosclerosis Risk in Communities Study, 1987-1998. Models were adjusted for race-center and education; age, BMI, cigarette smoking status, alcohol beverage consumption status, and antihypertensives at time 2; and elapsed time between visits. *Significantly different $(P<0.05)$ from weight maintainers. 

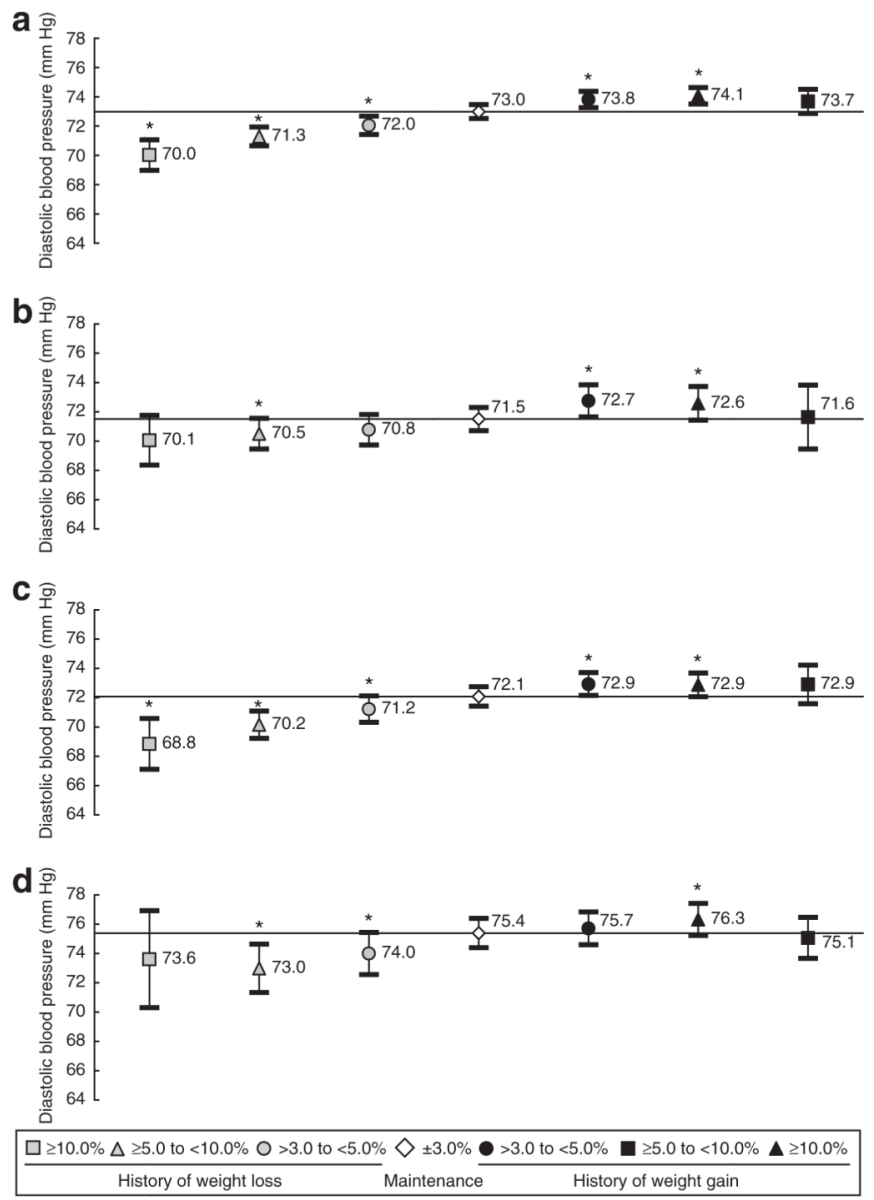

Figure 4.

Current mean diastolic blood pressure (and 95\% confidence intervals) among men with different 3-year weight histories, (a) overall and by current weight status ((b) normal weight; (c) overweight; or (d) obese), the Atherosclerosis Risk in Communities Study, 1987-1998. Models were adjusted for race-center and education; age, BMI, cigarette smoking status, alcohol beverage consumption status, and antihypertensives at time 2; and elapsed time between visits. *Significantly different $(P<0.05)$ from weight maintainers. 


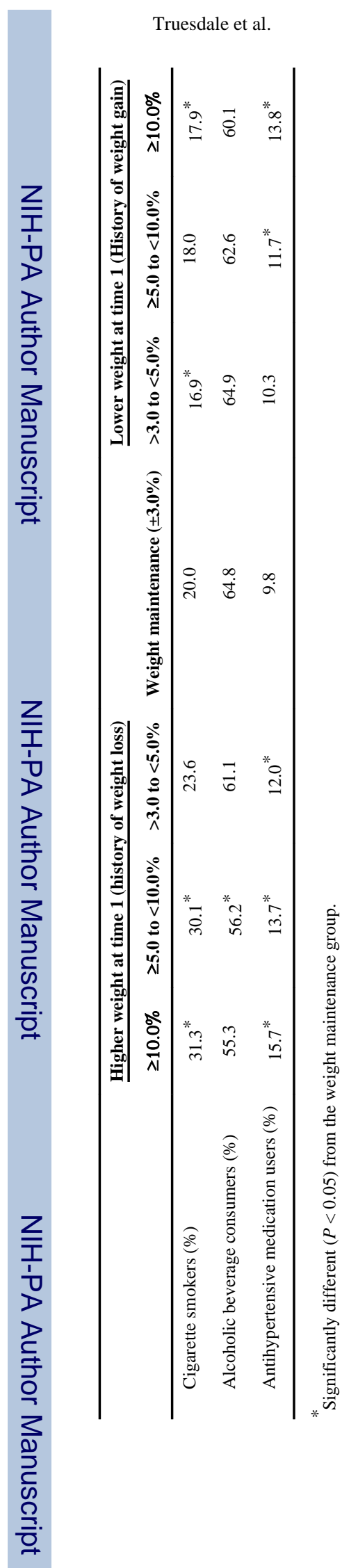

Obesity (Silver Spring). Author manuscript; available in PMC 2011 December 9. 
Truesdale et al.

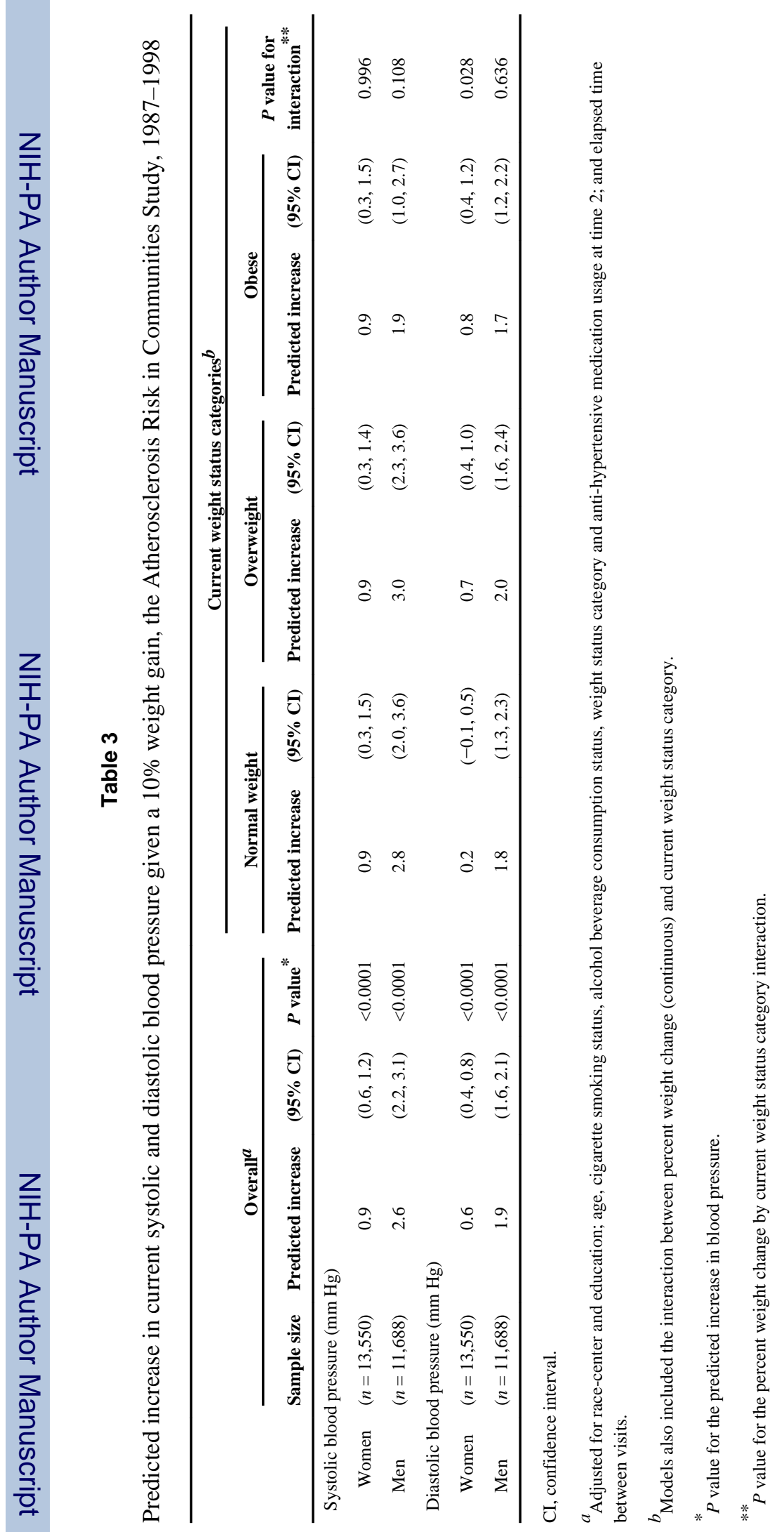

Obesity (Silver Spring). Author manuscript; available in PMC 2011 December 9. 